\title{
Two-Dimensional Magnetotransport in the Extreme Quantum Limit
}

\author{
D. C. Tsui, (a), (b) H. L. Stormer, (a) and A. C. Gossard \\ Bell Laboratories, Murray Hill, New Jersey 07974 \\ (Received 5 March 1982)
}

\begin{abstract}
A quantized Hall plateau of $\rho_{x y}=3 h / e^{2}$, accompanied by a minimum in $\rho_{x x}$, was observed at $T<5 \mathrm{~K}$ in magnetotransport of high-mobility, two-dimensional electrons, when the lowest-energy, spin-polarized Landau level is $\frac{1}{3}$ filled. The formation of a Wigner solid or charge-density-wave state with triangular symmetry is suggested as a possible explanation.

PACS numbers: 72.20.My, 71.45.-d, 73.40.Lq, 73.60.Fw
\end{abstract}

In the presence of an intense perpendicular magnetic field $B$, a system of two-dimensional (2D) electrons is expected to form a Wigner sol$\mathrm{id}^{1,2}$ at low temperatures $(T)$. In the infinite $-B$ limit, an analogy can be drawn to the classical electron gas on the surface of liquid helium, which crystallizes into a solid ${ }^{3}$ when the ratio of the electron's average potential energy to thermal energy $\Gamma \equiv e^{2} \pi^{1 / 2} n / \epsilon k T=137$ ( $n$ is the electron areal density). At finite $B$, quantum effects become important and it has been suggested that a charge-density-wave (CDW) state ${ }^{4}$ may be possible at considerably higher $T$ as a precursor to Wigner crystallization. Early experiments were carried out on the Si inversion layer at the $\mathrm{Si}-$ $\mathrm{SiO}_{2}$ interface. Kawaji and Wakabayashi ${ }^{5}$ and Tsui $^{6}$ made high- $B$ magnetoconductivity measurements and observed structures and electric field dependences which cannot be explained by the independent-electron theory of Ando and Uemura. ${ }^{7}$ Subsequently, Kennedy et $a l .{ }^{8}$ observed a shift in cyclotron resonance, concomitant with a drastic line narrowing, in the high- $B$ limit, when the average electron separation exceeds the cyclotron diameter. Wilson, Allen, and Tsui ${ }^{9}$ studied the dependence of this phenomenon on the Landaulevel filling factor $(\nu=n h / e B)$, and found that a pinned-CDW model ${ }^{10}$ gave the most satisfactory account of the cyclotron resonance data. However, in the range of $n$ at which these experiments were performed, localization due to disorder at the $\mathrm{Si}$ $\mathrm{SiO}_{2}$ interface is known to be important even in the absence of $B$, and consequently, it has not been possible to discern true Coulomb effects from those due to disorder.

In this Letter, we report some striking, new results on the transport of high-mobility, 2D electrons, in GaAs-AlGaAs heterojunctions, in the extreme quantum limit $(\nu<1)$, when the lowest-energy, spin-polarized Landau level is partially filled. We found that at temperatures $T<5$ $\mathrm{K}$, the diagonal part $\rho_{x x}$ of the resistivity tensor shows a dip at $\nu=\frac{1}{3}$, which becomes stronger at lower $T$. For $\nu<\frac{1}{3}, \rho_{x x}$ follows an approximately exponential increase with inverse $T$. The Hall resistivity $\rho_{x y}$, on the other hand, approaches a step of $3 h / e^{2}$ at $\nu=\frac{1}{3}$ as $T$ decreases, but remains essentially independent of $T$ away from this Hall plateau. These features of the data resemble those of the quantized Hall resistance and the zero-resistance state expected exclusively for integral values of $\nu$. We suggest that these striking results are evidence for a new electronic state at $\nu=\frac{1}{3}$. They are consistent with the notion that a Wigner solid, or a CDW state with triangular crystal symmetry, is favored at $\nu=\frac{1}{3}$ when the unit cell area of the lattice is a multiple of the area of a magnetic flux quantum.

The samples, consisting of $1-\mu m$ undoped GaAs, 500- $\AA$ undoped $\mathrm{Al}_{0.3} \mathrm{Ga}_{0.7} \mathrm{As}, 600-\AA$ Si-doped $\mathrm{Al}_{0.3} \mathrm{Ga}_{0.7} \mathrm{As}$, and 200- $\mathrm{A}$ Si-doped GaAs single crystals, were sequentially grown on insulating GaAs substrates using molecular-beam-epitaxy techniques. ${ }^{11}$ The 2D electron gas, resulting from ionized donors placed $500 \AA$ inside AlGaAs, ${ }^{12}$ is established at the undoped GaAs side of the GaAsAlGaAs heterojunction. Samples were cut into standard Hall bridges and Ohmic contacts to the electron layer were made with In at $400^{\circ} \mathrm{C}$. Lowfield transport measurements were used to determine $n$ and $\mu$. Our samples have $n$ from $1.1 \times 10^{11}$ to $1.4 \times 10^{11} \mathrm{~cm}^{-2}$ and $\mu$ from 80000 to 100000 $\mathrm{cm}^{2} / \mathrm{V}$ sec. The high- $B$ measurements were performed at the Francis Bitter National Magnet Laboratory, Cambridge, Mass.

Figure 1 shows $\rho_{x y}$ and $\rho_{x x}$ of one specimen as a function of $B$ at four different temperatures. The scale at the top of the figure shows the Landau level filling factor $\nu$, which gives the number of occupied levels. At integral values of $\nu$, the data show the characteristic features of the quantized Hall plateaus and the vanishing of $\rho_{x x},{ }^{13-15}$ when the Fermi energy $E_{\mathrm{F}}$ is pinned in the gap between two adjacent levels. Removal of spin de- 


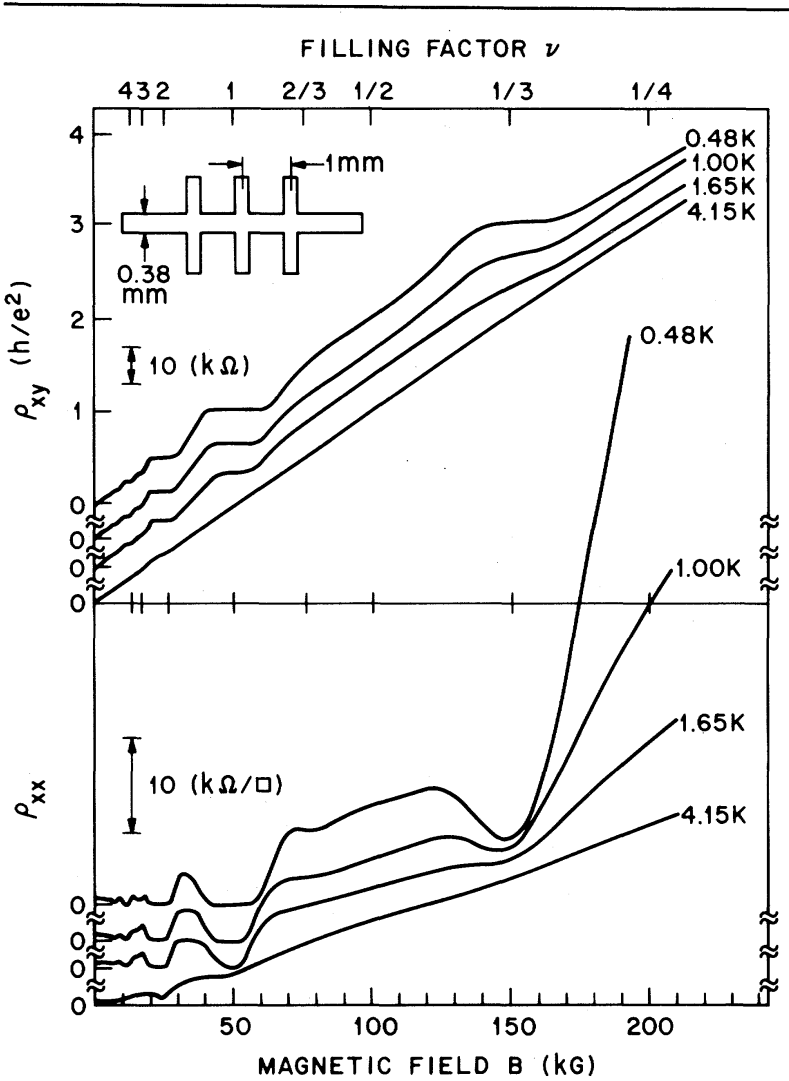

FIG. 1. $\rho_{x y}$ and $\rho_{x x}$ vs $B$, taken from a $\mathrm{GaAs}-\mathrm{Al}_{0.3^{-}}$ $\mathrm{Ga}_{0.7}$ As sample with $n=1.23 \times 10^{11} / \mathrm{cm}^{2}, \mu=90000 \mathrm{~cm}^{2} /$ $\mathrm{V}$ sec, using $I=1 \mu \mathrm{A}$. The Landau level filling factor is defined by $\nu=n h / e B$.

generacy ${ }^{16}$ is seen in the appearance of these features at odd-integer values of $\nu$. As observed earlier, ${ }^{17}$ the plateaus in $\rho_{x y}$ as well as the vanishing of $\rho_{x x}$ become increasingly pronounced as $T$ is decreased.

In the extreme quantum limit, $\nu<1$, only the lower spin state of the lowest Landau level, i.e., the $(0, \uparrow)$ level, remains partially occupied. In this regime (i.e., $B>50 \mathrm{kG}$ in Fig. 1), the sys tem is completely spin polarized. For $T>4.2 \mathrm{~K}$, $\rho_{x y}=B / n e$, and $\rho_{x x}$ shows also nearly linear dependence on $B$, as expected from the free-electron theory of Ando and Uemura. ${ }^{7,16}$ At lower $T$, $\rho_{x y}$ deviates from $\rho_{x y}=B / n e$ at $\nu \sim \frac{1}{3}$. This deviation becomes more pronounced as $T$ decreases and approaches a plateau of $\rho_{x y}=3 h / e^{2}$, within an accuracy better than $1 \%$ at $0.42 \mathrm{~K}$. The appearance of this plateau is accompanied by a minimum in $\rho_{x x}$, as apparent in the lower panel of Fig. 1. The development of these features is similar to that of the quantized Hall resistance and the concomitant vanishing of $\rho_{x x}$, observed at integral

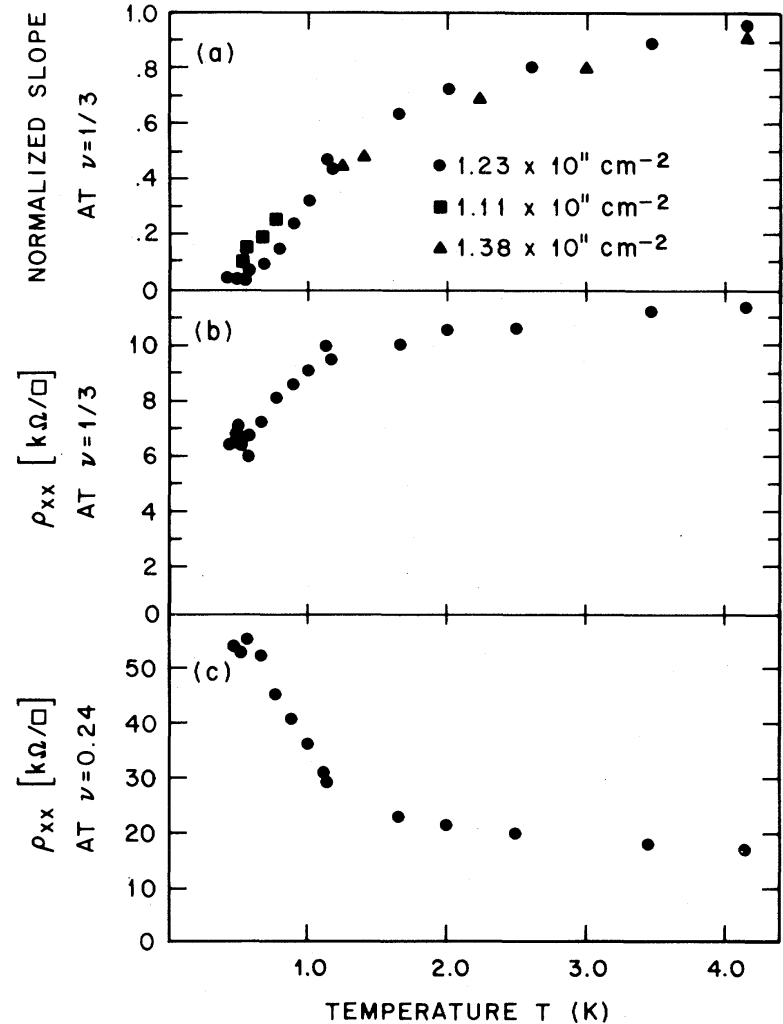

FIG. 2. $T$ dependence of (a) the slope of $\rho_{x y}$ at $\nu=\frac{1}{3}$, normalized to the slope at $\sim 30 \mathrm{~K}$, (b) $\rho_{x x}$ at $\nu=\frac{1}{3}$, and (c) $\rho_{x x}$ at $\nu=0.24$.

values of $\nu$ at higher $T$. Moreover, for $\nu<\frac{1}{3}$ and away from the plateau region, $\rho_{x x}$ shows strong increase with decreasing $T$, while $\rho_{x y}$ shows very weak decrease or essentially independence of $T$. This behavior has been seen to $\nu=0.21$, the smallest $\nu$ attained in this experiment.

Figure 2 illustrates the development of $\rho_{x x}$ and $\rho_{x y}$ at fixed $B$ as a function of $T$. Figure 2(a) shows the slope of $\rho_{x y}$ at $\nu=\frac{1}{3}$, normalized to the slope at high $T(\sim 30 \mathrm{~K})$, for three samples with slightly different $n$. Figure 2(b) shows the accompanying $\rho_{x x}$ minimum (at $\nu=\frac{1}{3}$ ), and Fig. 2(c) shows $\rho_{x x}$ at $\nu=0.24$ to illustrate its $T$ dependence for $\nu<\frac{1}{3}$, away from the Hall plateau. Several points should be noted. First, the slope of $\rho_{x y}$ at $\nu=\frac{1}{3}$ approaches zero at $T \sim 0.4 \mathrm{~K}$, indicative of a true quantized Hall plateau. Second, replotting the data in Fig. 2(a) on logarithmic slope versus inverse $T$ scale shows a linear portion for data taken at $T \geqslant 1.1 \mathrm{~K}$. This fact allows us to extrapolate the normalized slope to 1 at $T_{0}=5 \mathrm{~K}$, which we identify as the temperature for the onset of this phenomenon. Third, $\rho_{x x}$ at $\nu=\frac{1}{3}$ is $\sim 6 \mathrm{k} \Omega / \square$ 
at our lowest $T$ of $\sim 0.42 \mathrm{~K}$, and much lower $T$ is needed to determine if $\rho_{x x}$ at $\nu=\frac{1}{3}$ indeed vanishes with vanishing $T$. Finally, if the data for $\rho_{x x}$ at $\nu=0.24$ [Fig. 2(c)] are replotted on logarithmic $\rho_{x x}$ versus inverse $T$ scale, an exponential dependence on $1 / T$ is seen for $T \geqslant 0.6 \mathrm{~K}$, with a preexponential factor of $\sim 13 \mathrm{k} \Omega / \square$. This result may be interpreted as due to thermally activated transport with an activation energy of $0.94 \mathrm{~K}$. The preexponential factor is considerably lower than the maximum metallic sheet resistance of $\sim 40 \mathrm{k} \Omega /$ $\square$, predicted for Anderson localization in the tails of Landau levels, ${ }^{18}$ but comparable to that signifying a metal-insulator transition for $2 \mathrm{D}$ systems in the absence of $B .^{19}$ Moreover, the data for $\nu<\frac{1}{3}$ suggest a state of localization, in which the electron mobility is thermally activated, ${ }^{17}$ as seen in $\rho_{x x}$. The electron density, as seen in the slope of $\rho_{x y}$ vs $B$, remains essentially independent of $T$.

The existence of quantized Hall resistance accompanied by the vanishing of $\rho_{x x}$ at integral values of $\nu$ is now well known. Their observation is attributed to the existence of an energy gap between the extended states in two adjacent Landau levels and the presence of localized states, which pin $E_{\mathrm{F}}$ in the gap, to keep all the extended states in finite numbers of Landau levels completely occupied for finite regions of $B$ or $n$. Laughlin's argument ${ }^{20}$ based on gauge invariance demonstrated that the quantized Hall resistance, given by $\rho_{x y}=h / i e^{2}(i=1,2, \ldots)$, results from complete occupation of all the extended states in the Landau level, regardless of the presence of localization. Our observation of a quantized Hall resistance of $3 h / e^{2}$ at $\nu=\frac{1}{3}$ is a case where Laughlin's argument breaks down. If we attribute it to the presence of a gap at $E_{\mathrm{F}}$ when $\frac{1}{3}$ of the lowest Landau level is occupied, his argument will lead to quasiparticles with fractional electronic charge of $\frac{1}{3}$, as has been suggested for $\frac{1}{3}$-filled quasi one-dimensional systems. ${ }^{21}$

At the present, there is no satisfactory explanation for all of our observations. The fact that this phenomenon always occurs at $\nu=\frac{1}{3}$ and that it is most striking in samples with the highest electron mobility suggest the formation of a new spin-polarized electronic state, such as Wigner solid or CDW, with a triangular symmetry, ${ }^{22}$ which is favored at $\nu=\frac{1}{3}$. In this picture, the observed features of $\rho_{x x}$ and $\rho_{x y}$ may be attributed to transport of the collective ground state. At $T=0$, the transport is free of dissipation and $\rho_{x x}$ is expected to vanish. Since the number of electrons in this ground state is $n=e B / 3 h$, the Hall resistivity is $\rho_{x y}=B / n e=3 h / e^{2}$. As discussed by Baraff and Tsui, ${ }^{23}$ observation of the quantized Hall plateau may be attributed to the presence of donor states inside $\mathrm{Al}_{x} \mathrm{Ga}_{1-x} \mathrm{As}$. The thermal activation of $\rho_{x x}$ at $\nu=\frac{1}{3}$ may result from activation of defects in the condensate, which give rise to dissipation.

Finally, our data also show weaker, but similar, structures in $\rho_{x x}$ near $\nu=\frac{2}{3}$ and near $\nu=\frac{3}{2}$, accompanied by slight changes in the slope of $\rho_{x y}$. These structures, though discernible in Fig. 1, are well resolved in the data taken from the sample with $n=1.4 \times 10^{11} / \mathrm{cm}^{2}$ and $\mu=100000 \mathrm{~cm}^{2} / \mathrm{V}$ sec at $1.2 \mathrm{~K}$. In our picture, the structure at $\nu$ $=\frac{2}{3}$ may be identified as due to the formation of a Wigner solid or CDW of holes, expected from electron-hole symmetry. At $\nu=\frac{3}{2}$, the $(0, \uparrow)$ Landau level is completely filled, but the $(0, \downarrow)$ level is half filled. Consequently, $\frac{2}{3}$ of all the electrons occupy the low-lying $(0, \uparrow)$ level, and only the remaining $\frac{1}{3}$ with spin $\downarrow$ may participate in the formation of a collective ground state. Our data appear to suggest that this condition is also favored for a Wigner solid or CDW ground state.

In summary, we observed striking structures in the magnetotransport coefficients of high- $\mu$, $2 \mathrm{D}$ electrons in $\mathrm{GaAs}-\mathrm{Al}_{x} \mathrm{Ga}_{1-x} \mathrm{As}$ heterojunctions at $\nu=\frac{1}{3}$, and similar, but much weaker, structures at $\nu=\frac{2}{3}$ and $\frac{3}{2}$. Their development as a function of $T$ is reminiscent of the quantized $\rho_{x y}$ and the concomitant vanishing of $\rho_{x x}$, expected only for integral values of $\nu$. We suggest as a possible explanation the formation of a new electronic state, such as a Wigner solid or CDW state with a triangular symmetry.

We thank P. M. Tedrow for the $\mathrm{He}^{3}$ refrigerator; R. B. Laughlin, P. A. Lee, V. Narayanamurti, and P. M. Platzman for discussions; and K. Baldwin, G. Kaminsky, and W. Wiegmann for technical assistance. This work was supported in part by the National Science Foundation.

\footnotetext{
${ }^{(a)}$ Visiting scientist at the Francis Bitter National Magnet Laboratory, Cambridge, Mass. 02139.

${ }^{(b)}$ Present address: Department of Electrical Engineering and Computer Science, Princeton University, Princeton, N.J. 08544.

${ }^{1}$ E. P. Wigner, Phys. Rev. $\underline{46}, 1002$ (1934).

${ }^{2}$ Y. E. Lozovik and V. I. Yudson, Pis'ma Zh. Eksp. Teor. Fiz. 22 26 (1975) [JETP Lett. 22, 11 (1975)].

${ }^{3}$ C. C. Grimes and G. Adams, Phys. Rev. Lett. $\underline{42}$, 795 (1979).

${ }^{4}$ H. Fukuyama, P. M. Platzman, and P. W. Anderson, Phys. Rev. B $\underline{19}, 5211$ (1979).
} 
${ }^{5}$ S. Kawaji and J. Wakabayashi, Solid State Commun. $\underline{22}, 87$ (1977).

${ }^{6}$ D. C. Tsui, Solid State Commun. 21, 675 (1977).

${ }^{7}$ T. Ando and Y. Uemura, J. Phys. Soc. Jpn. $\underline{36}, 959$ (1974) .

${ }^{8}$ T. A. Kennedy, R. J. Wagner, B. D. McCombe, and D. C. Tsui, Solid State Commun。22, 459 (1977)。

${ }^{9}$ B. A. Wilson, S. J. Allen, and D. C. Tsui, Phys. Rev. Lett. 44 , 479 (1980).

${ }^{10}$ H. Fukuyama and P. A. Lee, Phys. Rev. B $\underline{18}, 6245$ (1978) .

${ }^{11}$ A. Y. Cho and J.R. Arthur, Prog. Solid State Chem. 10,157 (1975).

${ }^{12}$ H. L. Stormer, A. Pinczuk, A. C. Gossard, and W. Wiegmann, Appl. Phys. Lett. 38, 691 (1981); T. J. Drummond, H. Morkoc, and A. Y. Cho, J. Appl. Phys. 52, 1380 (1981).

${ }^{13}$ K. von Klitzing, G. Dorda, and M. Pepper, Phys. Rev. Lett. $\underline{45}, 494$ (1980).

${ }^{14}$ D. C. Tsui and A. C. Gossard, Appl. Phys. Lett. 37,

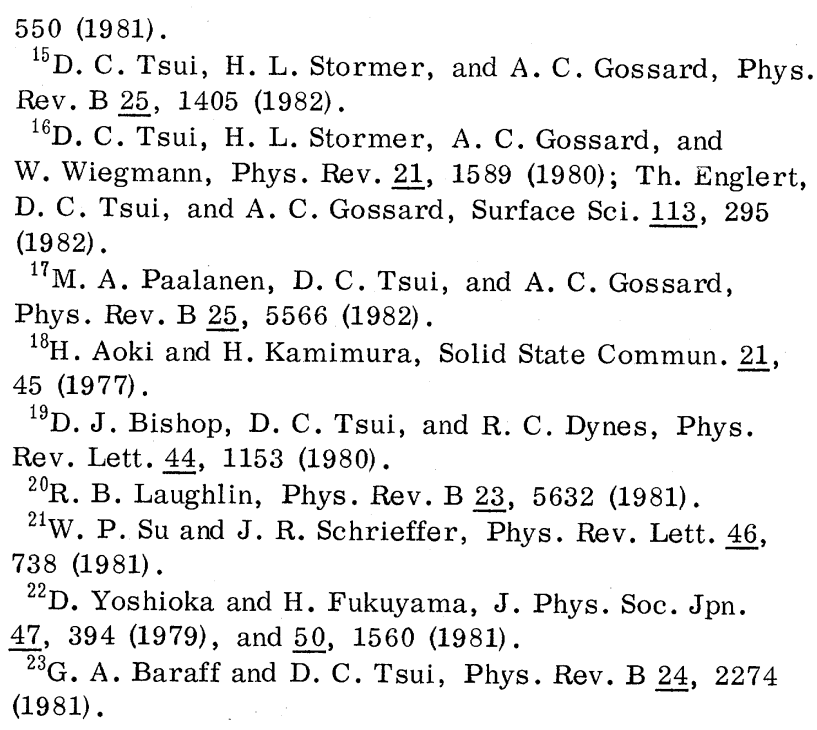

\title{
Test of High- $p_{T}$ Scaling from Cosmic-Ray Interactions up to $400 \mathrm{TeV}$
}

\author{
W. E. Hazen and A. Z. Hendel \\ Randall Laboratory of Physics, University of Michigan, Ann Arbor, Michigan 48109 \\ and \\ A. G. Ash, J. M. Foster, A. L. Hodson, and M. R. Porter \\ Department of Physics, University of Leeds, Leeds, United Kingdom \\ and \\ R. M. Bull \\ Department of Physics, University of Nottingham, Nottingham, United Kingdom \\ (Received 19 April 1982)
}

\begin{abstract}
The rate of subcores in cosmic-ray air showers has been measured near sea level with a close-packed $35-\mathrm{m}^{2}$ array of spark chambers at Leeds. Auxiliary experiments demonstrate that our transition effects are negligible. The rate of subcores versus shower size is translated into the rate of high- $p_{T}$ events versus energy of the interacting hadron. Comparison with calculations of Halzen favors $p_{T}{ }^{-4}$ over $p_{T}{ }^{-8}$ scaling. The highest energy bin is centered on $350 \mathrm{TeV}$.

PACS numbers: $94.40 . \mathrm{Rc}$
\end{abstract}

Cosmic rays provide a hadron beam with intensity sufficient for exploratory study of interactions up to energies of a few $100 \mathrm{TeV}$, that is, ten times higher than the energy from the super proton synchrotron collider at CERN. Therefore, the results can still be of interest in spite of the limit to accuracy inherent in the cosmicray method.

This is a report of an experiment ${ }^{1}$ to test models for deep-inelastic interactions by measuring the energy dependence of the cross section for high $-p_{T}$ production. The results are obtained from the observed rate of subcores in cosmicray air showers.
In view of the conflict in results among previous experiments, ${ }^{2}$ we first review the case in favor of our results. We then describe the analysis of our data and compare to Halzen's ${ }^{3}$ scaling from accelerator data. The results strongly favor the parton model with $p_{T}^{-4}$ scaling.

In order to interpret any subcore data, a connection must be made between high-energy secondary hadrons produced in the atmosphere and the subcore as observed in a detector. The information used to link the two comes either from computer simulations, ${ }^{4}$ or from analytical solutions of cascade shower equations, ${ }^{5}$ or from a combination. These have been carried out for 